\title{
Ambiguity in multicriteria quality decisions ${ }^{\text {in }}$
}

\author{
Isabel Gutiérrez*, Salvador Carmona
}

Universidad Carlos III, C/Madrid, 126, 28903 Getafe Madrid, Spain

\begin{abstract}
Quality is becoming an issue of increasing strategic importance in business. The aim of this paper is to analyze quality from a decision-making perspective. Quality decisions are characterized by their ambiguity while their evaluation uses a multicriteria viewpoint. Fuzzy decision theory provides a conceptual framework to model decisions with these features. It enables the decision maker to add his/her own experience and any other type of information to that obtained from hard figures. This theory is applied to a set of quality decision alternatives which are evaluated using different criteria such as their impact on fixed costs, cost of quality, leadtime and flexibility. The approach provided in this paper can be extended to other quality decisions.
\end{abstract}

Key words: Quality decision-making; Quality dimensions; Fuzzy multicriteria decision making

\section{Introduction}

Quality has become an important linchpin in the design and implementation of the organizational strategy. In this regard, one can establish a hierarchy of organizational strategies: corporate strategies, business unit strategies and functional strategies [1]. Although quality pervades the whole organization and every organizational activity [2], it may also be analyzed from its traditional manufacturing aspect. Manufacturing strategies are

\footnotetext{
"This paper is partially funded by the CICYT (Spain) grant SEC 930835 CO2 02 and the DGCYT (Spain) grant PB93-0233. We wish to thank Maria José Álvarez, Hanno Roberts, and two anonymous reviewers for their constructive suggestions on earlier drafts of this paper.

* Corresponding author. Fax: + 34.1.624 9608. E-mail: isagut@eco.uc3m.es.
}

functional strategies which embrace four main dimensions: cost, quality, flexibility and dependability [3]. The quality dimension is characterized by its ambiguity as well as by being subject to multiple criteria.

The definitions of ambiguity stress the ideas of imprecision and inexactness. Zadeh [4] pointed out that ambiguity has to do with classes of objects with no sharp or exact boundaries between what "is" and what "is not". In the particular case of quality, it is not always clear when a given product or service "has" quality; that is, quality has more to do with dimensions like "more" or "less" than with others such as "has" or "has not".

Reeves and Bednar [5] have developed an extensive review of the different definitions of quality. Quality definitions were classified into four main categories: quality as excellence, quality as value, quality as conformance to specifications and 
quality as meeting and/or exceeding customer expectations. After analyzing the strengths and weakenesses of these definitions, Reeves and Bednar [5] conclude that quality as excellence provides little practical guidance to practitioners as well as that it has measurement difficulties. Quality as value cannot extract the individual judgment while quality as exceeding and/or meeting customer expectations is difficult to measure; customers may not know their own expectations and there is a confusion between customer service and customer satisfaction. Finally, quality as conformance to specifications is inappropriate for services. Moreover, it potentially reduces organizational adaptability and consumers do not know or care about internal specifications.

The above three definitions of quality (excellence, value and exceeding and/or customer expectations) contain measurement problems, it being impossible to clearly establish what is quality and what is not.

The fourth definition (conformance to specifications) is unable to cope with the growing importance of services and is too much focused on internal specifications. Customers do not perceive individual product specifications but they form an overall judgment. The summation of each internal specification of the product is different from the overall perception formed by the customer.

Hence, quality is not a clear-cut concept but an ambiguous one. The definition of quality as exceeding and/or meeting customer's needs is widely accepted $[6,7]$ and, therefore, it will be used in the rest of this paper.

The meaning of "good" and "bad" quality is questionable $[8,9]$. In fact, quality decisions can be described as being "more or less correct" at best, and the "more or less" qualifier depends on the specific situation that the firm is experiencing at any given moment. Far from being straightforward decisions, quality decisions require the active participation of the decision-makers whose attitudes towards risk, personal values, teamwork, etc. are brought into the decision process. For this reason, quality decisions share the ambiguity that characterizes other business decisions such a product mix decisions [10], scheduling of flexible manufacturing systems [11], or the investigation of cost variances [12]. To deal with the problem of ambiguity the theory of fuzzy sets provides us with an adequate conceptual framework [13].

Quality decisions also require to be analyzed from a multiple criteria approach. Quality is no longer an isolated, independent function, dominated by technical experts [14]. Quality management is a multidisciplinary task carried out by the top management [15]. In this respect, the management team should take into account aspects that are far from the technical side of quality, like differences in societal structures or incentive systems [16]. These different perspectives justify that some actions on quality like mass inspection, while severely criticized in the literature [17], are still being sometimes implemented in cases where the customer security is at stake (i.e., when quality problems are found in the suspension system of motor vehicles).

As far as quality decisions are concerned, authors point out different dimensions of quality related to quality measurements [18]. Although quality literature has devoted a remarkable effort to determining the dimensions of quality and their related measurements, there is a lack of studies about the impact of the other dimensions of the manufacturing strategy on quality decisions. With few exceptions [19], by reviewing the literature one could almost be led to thinking that quality decisions are made in a vacuum, isolated from the other dimensions of the manufacturing strategy. This lack of references is even more remarkable when placed in a general context full of quotations about the (positive) impact of quality actions on the other dimensions of the manufacturing strategy: dimensions such as cost $[6,20]$, market share $[21,22]$, productivity $[21,23]$ or throughput time reduction [22].

The alternatives for improving quality are judged along different decision-making criteria. Although this aspect is widely recognized in the literature $[14,9]$, we have already mentioned that the relationship between quality decisions and other dimensions of the manufacturing process has not been analyzed from a decision-making point of view. This lack of theoretical studies is in sharp contrast with practices which link quality decisions with the remaining dimensions of the manufacturing strategy [19]. In the rest of the paper, the multiple criteria of quality decisions are related to 
their role within the strategic process. Further, it is precisely this multiple valuation which can offer us an explanation as to why some (bad) practices (i.e., mass inspection) are sometimes carried out in different companies. According to the criterion of "customer satisfaction" any potential danger to customer security could bring about mass inspections. Given that the fuzzy set theory is a suitable framework to analyze ambiguous decisions, a fuzzy multicriteria approach is the methodology chosen to model quality decisions.

The aim of this paper is to analyze quality from a decision-making perspective. We consider that two features pervade the quality decision itself: its ambiguity and its multiple criteria nature.

The rest of the paper is organized as follows: Section 2 briefly describes decision theory in fuzzy environments. Section 3 presents a fuzzy multicriteria model for quality decisions. Section 4 develops an application of the model and finally, Section 5 contains some concluding remarks.

\section{A review of the fuzzy decision theory}

The phenomenon of ambiguity and vagueness has been observed in many scientific disciplines. Since 1965, Zadeh has developed a research trend which states that fuzziness is the biggest source of imprecision in human systems, that is, in those systems that deal with human interaction. Because of this, Zadeh points out that conventional techniques applied to human systems when modelling imprecisions are frequently inadequate. These techniques require an accuracy level in the estimates which is often difficult to reach.

To explain the distinction between fuzziness and randomness and therefore to reduce the necessity of processing numerical inputs in decision analysis models, Zadeh [4] introduces the concept of fuzzy set:

Let $X$ be a space of points (objects), with a generic element denoted by $x$. Thus, $X=\{x\}$. A fuzzy set (class) $A$ in $X$ is characterized by a membership (characteristic) function $\mu_{A}(x)$ which associates with each point in $X$ a real number in the interval $[0,1]$, with the value of
$\mu_{A}(x)$ at $x$ representing the 'grade of membership' of $x$ in $A$.

Due to this, given a fuzzy set $A$, any member of $X$ can belong to it a "a little", "a lot", "intensely", etc. So, to define a fuzzy set correctly it is necessary to use pairs of values where the first value $x$ stands for an element of the set under consideration and the second value stands for the intensity with which that element belongs to the set

$A=\left\{\left(x, \mu_{A}(x)\right)\right\}$.

The membership function is a basic concept in fuzzy set theory. While in conventional or ordinary sets the characteristic function can only admit zero and one as values, in the membership functions for fuzzy sets admit values that belong to the closed interval $[0,1]$. This difference in the membership function is the main distinction between fuzzy and ordinary sets. Fuzzy set theory allows for gradual membership.

Basic operations among fuzzy sets used in this article are union and intersection. Zadeh [4] defined these operations as follows:

If $X$ is the universal set and $A$ and $B$ are two fuzzy subsets of $X$, with membership functions $\mu_{A}(x)$ and $\mu_{B}(x)$ :

(a) The union between $A$ and $B, A \cup B$, is defined as the fuzzy set $C$, such as

$$
\begin{aligned}
\mu_{C}(x) & =\mu_{A \cup B}(x) \\
& =\left[\mu_{A}(x), \mu_{B}(x)\right]=\mu_{A}(x) \vee \mu_{B}(x)
\end{aligned}
$$

with $\vee$ representing the maximum.

(b) The intersection between $A$ and $B, A \cap B$, will be another fuzzy set $D$ with the following membership function:

$$
\begin{aligned}
\mu_{D}(x) & =\mu_{A \cap B}(x) \\
& =\operatorname{Min}\left[\mu_{A}(x), \mu_{B}(x)\right]=\mu_{A}(x) \wedge \mu_{B}(x),
\end{aligned}
$$

$\wedge$ being the minimum.

The operators (connectives) Max and Min represent the logic operations "or" and "and". Gupta and Qi [24] stated that Zadeh's conventional operators, Min and Max, have been used in almost every design of fuzzy logic controllers and even in the modelling of other decision-making processes. 
However, some theoretical and experimental studies seem to indicate that other types of operators may work better in some situations. For instance, the product operator may be preferred to the Min operator [25].

The triangular norm (t-norm) and the triangular conorm (t-conorm) originated from the studies of probabilistic metric spaces were introduced into fuzzy set theory [26]. It is suggested that t-norm and $t$-conorm can be used for the intersection and union of fuzzy sets.

\section{Fuzzy decision-making framework}

Using the fuzzy sets theory, Bellman and Zadeh [27] propose a new conceptual framework for decision making. These authors define decision in fuzzy environments as the confluence of goals and constraints. Furthermore, both goals and constraints are treated symmetrically. The basis of decision making in fuzzy environments is characterized by considering goals and constraints as fuzzy sets in the space of alternatives. Therefore, these alternatives can be treated identically during the decisionmaking process. More specifically, let $X=\{x\}$ be a given set of alternatives.

If $G$ is a fuzzy goal with a membership function $\mu_{G}(x)$ and $C$ a fuzzy constraint with a membership function $\mu_{C}(x)$, then $D$ is a fuzzy decision which results from the intersetion of $G$ and $C$ with a membership function

$\mu_{D}(x)=\mu_{G}(x) \wedge \mu_{C}(x)$.

In general terms, if there are $m$ goals $G_{1}$, $G_{2}, \ldots, G_{m}$, and $n$ constraints, $C_{1}, C_{2}, \ldots, C_{n}$, the resulting decision set will be the fuzzy set

$D=G_{1} \cap G_{2} \cap \cdots G_{m} \cap C_{1} \cap C_{2} \cap \cdots \cap C_{n}$

with membership function

$$
\begin{gathered}
\mu_{D}(x)=\operatorname{Min}\left[\mu_{G_{1}}(x), \mu_{G_{2}}(x), \ldots, \mu_{G_{m}}(x),\right. \\
\left.\mu_{C_{1}}(x), \ldots, \mu_{C_{n}}(x)\right] .
\end{gathered}
$$

Given set $D$ one can obtain an optimal decision, $x_{0}$ :

$$
\mu_{D}\left(x_{\mathrm{o}}\right)=\max \mu_{D}(x)=\bigvee \mu_{D}(x)
$$

i.e., is the optimal decision is an alternative which maximizes the membership function of the fuzzy set.

Min and Max operators shown in Eqs. (6) and (7) may be substituted by other connectives as t-norms and their related dual $t$-conorms.

Within this conceptual framework, several procedures which consider the problem of decision making under multiple criteria have been developed. In this case, all the criteria are connected by the intersection operator. The selected alternative is the element with the greater membership value in the intersection fuzzy subset. In this article this multicriteria approach is used to evaluate quality decisions.

\section{Fuzzy multicriteria analysis}

Decision theory was improved with the advances of multicriteria programming $[28,29]$. In the particular area of fuzzy decision-making, two major research areas have evolved: multiple-objective decision-making and multi-attribute decision-making [30]. While the focus of both research areas is decision-making with several criteria, the former area concentrates on continuous decision spaces, primarily on mathematical programming with several objective functions [31,32], and the latter area deals with problems in discrete decision spaces [33-36].

This paper draws upon the second approach since it can be adapted well to quality problems. More specifically, the Yager's [33] method is used for its fulfillment of quality decision problems.

The description of this quality decision problem is as follows:

Let $X=\left\{x_{1}, x_{2}, \ldots, x_{i}, \ldots, x_{n}\right\}$ be a set of alternatives and let $C=\left\{C_{1}, C_{2}, \ldots, C_{j}, \ldots, C_{m}\right\}$ be a set of decision criteria.

Yager [33] considers these criteria as fuzzy subsets in the space of alternatives, that is, the attainment criterion $C_{j}$ by alternative $x_{i}$ is expressed by its degree of membership $\mu_{C_{i}}\left(x_{i}\right)$. The set of possible decisions is given using (5) as the intersection of $C_{j}$.

If the criteria under consideration are of different relative importance to the decision, then it is necessary to weigh them by their importance. If $w_{j}$ 
represents the importance of criteria $j$, the fuzzy decision set is then given by

$D=C_{1}^{w_{1}} \cap C_{2}^{\omega_{2}} \cap \cdots \cap C_{m}^{w_{m}}$

where $C_{j}^{w_{j}}$ is a fuzzy subset with the membership function

$\left[\mu_{\mathrm{C}_{j}}\left(x_{i}\right)\right]^{w_{j}}$.

To determine the values of $w_{j}$, Yager suggests Saaty's hierarchical procedure (AHP) for determining weights by computing the eigenvectors of the matrix $M$ of relative weights of subjective estimates [37]. The method proposed by Saaty [38] is based on a matrix of pairwise comparisons and eigenvalue theory. AHP has been revised by Federov et al. [39] and Triantaphyllov and Mann [40] among others. Applications and revisions of AHP have not been exempt of severe criticisms [41,42]. Nevertheless, a number of researchers still consider AHP a useful procedure to rank human preferences $[43,44]$.

According to Saaty, given $m$ criteria it is possible to build a scale which permits their evaluation depending upon their importance for the decision. To carry out this process, the decision-maker has to make a series of pairwise comparisons. Thus, when criterion $C_{j}$ is compared with $C_{k}$ the values $b_{j k}$ and $b_{k j}$ are assigned in the following way:

(1) $b_{j k}=1 / b_{k j}$.

(2) If $C_{j}$ is more important than $C_{k}$, then $b_{j k}$ is a value of the set $\{1,2, \ldots, 9\}$, where 1 shows equal importance between $C_{j}$ and $C_{k}$, and 9 indicates that $C_{j}$ is much more important than $C_{k}$.

The matrix $M(m \times m)$ with the following structure is obtained:

(1) $b_{j j}=1$.

(2) $b_{j k}, i \neq k$, is determined by pairwise comparison between criteria using the previously mentioned procedure. The rest of the matrix is calculated by the relation

$b_{j k}=1 / b_{k j}$.

Saaty has shown that the eigenvector corresponding to the maximum eigenvalue of $M$ is a cardinal scale for the elements being compared.
So, the eigenvector

$Q=\left[\begin{array}{c}q_{1} \\ q_{2} \\ \vdots \\ q_{m}\end{array}\right]$

which fulfills the condition

$M \times Q=k_{\max } \times Q$

with $k_{\max }$ as the maximum eigenvalue of $M$, defines a system of weighting coefficients such that

$\sum_{j=1}^{m} q_{j}=1$

To weigh the criteria, Yager [33] proposes the substitution of the unit eigenvector $Q$ for the vector $W$ which satisfies

$W=m \times Q=\left[\begin{array}{c}w_{1} \\ w_{2} \\ \vdots \\ w_{m}\end{array}\right]=\left[\begin{array}{c}m \cdot q_{1} \\ m \cdot q_{2} \\ \vdots \\ m \cdot q_{m}\end{array}\right]$,

$\sum_{j=1}^{m} w_{j}=m$.

Thus, the membership grades in criteria with little importance $(w<1)$ become larger, while the memberships grades of those that are more important $(w>1)$ become smaller.

The membership function of the fuzzy subset $D$ is

$$
\begin{gathered}
\mu_{D}\left(x_{i}\right)=\operatorname{Min}\left[\left(\mu_{C_{1}}\left(x_{i}\right)\right)^{w_{1}},\left(\mu_{C_{2}}\left(x_{i}\right)\right)^{w_{2}} \cdots\right. \\
\left.\left(\mu_{C_{3}}\left(x_{i}\right)\right)^{w_{m}}\right],
\end{gathered}
$$

and the alternative selected, $x_{0}$, is the alternative that satisfies Eq. (7).

\section{A decision model for quality}

Let us assume the example of a car components producing plant which needs to be modernized in order to become a certified supplier for major car manufacturers. The plant produces suspension systems using a batch production process. The 
product structure is basically convergent-divergent on a job order sytem. The purchasing policy employs the EOQ system. Nevertheless, the plant will be awarded a certified suppliership only if it adopts to the JIT philosophy already being implemented by its target customers. The performance of the supplier will be assessed in terms of quality, timely delivery, flexibility and cost. The plant has a low performance profile in quality. Equipment flexibility is presently very low. Hence, the plant considers the purchasing of new equipment for key manufacturing areas. The purchase of this equipment is to be matched by changes in the organizational structure, i.e., personnel training, an agressive preventive maintenance policy and a redesign of the quality inspection system.

There are many actions which have strong repercussions on quality and that influence any quality decisions, albeit indirectly. This would be the case of a general housekeeping program or of a general purchase of tools. These actions should have a positive impact on quality and as such, they are often considered during the quality decision-making process. Nevertheless, every decision-maker takes into account only a limited number of alternatives; one normally does not consider those alternatives with indirect effects such as those under the strict control of other functional areas. Likewise, the decisionmaker usually dismisses right away certain actions. This is the case of those actions which are against the company's general policy on personnel security or against environmental control regulations, even though they might end up being quality improving.

The managing director has delegated decision autonomy to the operations manager and his team to cope with the problem of adapting the plant the new manufacturing environment. Considering weak quality performance, the operations manager and his team assess all the action alternatives with respect to their quality impact. On the one hand, the operations manager faces ambiguity coming from the new and explicit quality policy of exceeding and/or meeting customers' expectations. He also has to trade-off quality actions both with financial constraints and with the pressure of getting some early results from the certified supplier program.
This is the concrete set of alternatives under the consideration of the operations manager and his team:

$x_{1}$ : purchasing new machinery. The purchase of new machinery is an expensive choice but it has much to do with quality policies. For instance, a capital-intensive company such as the Commercial Nuclear Fuel Division of Westinghouse has estimated that $75 \%$ of its capital allocations relate to quality [45].

$x_{2}:$ workforce training. This is a regular element in quality programs. For example, Motorola spent $\$ 170$ million ( $2.8 \%$ of payroll) on workforce quality training during the period 1986-1990 [45].

$x_{3}$ : preventive maintenance. This is one of the basic elements of the Japanese approach to quality [46].

$x_{4}$ : contracting quality programs with subsequent suppliers. Suppliers development through these kind of actions is a central part of any JIT implementation program. It aims both at quality assurance and leadtime reduction [47].

$x_{5}$ : inspection. The operations manager aims at a redesign of the inspection function since it is not a proper part of the organization. Considering that the plant manufactures suspension system, product features require a $100 \%$ automatic inspection of certain product dimensions. Additionally, full batch inspections are also needed when car security is at stake.

Operations management literature identifies four critical success factors of today's manufacturing environment: cost, flexibility, leadtime and quality (e.g., $[48$, p. $11 ; 49$, pp. 44-46]). For the purposes of this example, we will assume company's criteria are based upon the above four critical success factors:

$C_{1}$ : reduction of total costs. Total costs are classified into fixed and variable costs.

$C_{2}$ : flexibility increases. Flexibility is the firm's capacity to respond to the requirements of the market. The company is presently trying to obtain new contracts from major car manufacturers. Consequently, the company needs to improve its flexibility in order to be able to produce a larger product variety. As a result, the company would be able to supply products with different specifications as well as respond quickly to market demands. Flexibility will be measured by means of set-up time. 
$C_{3}:$ reduction of leadtime. Leadtime is defined as the time which passes between the reception of the customer's order to the moment in which it is delivered. Through the reduction of leadtime, the company will be able to simultaneously increase flexibility and to decrease total costs ceteris paribus the costs of quality. Total leadtime can be split up into two elements: raw materials supply leadtime and production leadtime.

The former can be reduced by means of a suppliers development program. The latter can be reduced by means investments in new technology and/or improvements in operations management.

$C_{4}$ : reduction of the cost of quality $(C O Q) . \mathrm{COQ}$ is already an old concept but its usage has stimulated recent important actualizations [50]. COQ is made up of four elements: prevention costs, appraisal costs, internal failure and external failure. Prevention costs include all preventive measures such as the editing of a quality manual or quality circles. Appraisal costs include activities such as laboratory tests. Internal costs refer to scraps and reworks. Finally, the external failure costs are the consequence of poor quality products shipped to customers. The theory of COQ establishes that for a given total level of COQ, a company should rather pay prevention costs than pay costs for external failure.

Although these are just four criteria and therefore they cannot embrace the whole range of possibilities, these particular criteria have been chosen because of their implications in the design and implementation of the manufacturing strategy. Considering these four criteria as a whole, one should notice their close relationship with the multiple dimensions of the manufacturing strategy as defined in terms of cost, quality, flexibility and dependability [3]. Despite this relationship, it should be pointed out that both the alternatives and the criteria should be considered as a limited choice which can be expanded. The evaluation of these decisions is carried out according to certain criteria. This evaluation considers those criteria as fuzzy subsets of the decisions and their membership functions reflect the degree with which each decision satisfies each specific criterion.

Because of this, each particular decision criterion, $C_{j}$, is evaluated according to the effect that each concrete decision has upon the decision- maker's satisfaction. The greater the decisionmaker's satisfaction with the effect, the higher the value of the membership function assigned to it. On the other hand, the decision maker will assign a membership function close or equal to 0 whenever the repercussion level is intolerable.

Returning to the evaluation criteria of the example mentioned above, it should be said that the decision-maker prefers small values of total costs, set-up time, leadtime and COQ. A zero value for these criteria would therefore be the ideal case, and the membership functions assigned by the decision-maker would, hence, be one. Regarding the values of total costs, set-up time, lead time and COQ increase, the decision-maker will consider that the situation is worsening. Therefore, the membership functions assigned to these four criteria will progressively decrease. Finally, the decision-maker will consider that total costs, set-up time, leadtime and COQ above a given level are just unacceptable by the company, assigning membership functions close or equal to zero.

As proposed by Bellman and Zadeh's [27] framework, criteria $\left(C_{j}\right)$ are at the same time operative constraints. In other words, a criterion such as reducing total costs also functions as a constraint to increase total costs. If this criterion is not met by a given alternative, $x_{i}$, the decision-maker will assign a value close or equal to 0 .

This definition of the decision criteria allows us to evaluate the different alternatives related to them. Thus, each criterion is represented by a fuzzy subset in the space of alternatives.

The assignment of membership values to the alternatives under consideration is shown below. In this regard, we will assume that the company is going through a period of financial restrictions that result in a trade-off between the need of short-term results and the resources available to become a certified supplier of major car manufacturers. Let us suppose, then, that the decision-maker assigns the following values:

$$
\begin{aligned}
& C_{1}=\left\{\left(x_{1}, 0.1\right)\left(x_{2}, 0.8\right)\left(x_{3}, 0.3\right)\left(x_{4}, 0.5\right)\left(x_{5}, 0.3\right)\right\}, \\
& C_{2}=\left\{\left(x_{1}, 0.8\right)\left(x_{2}, 0.6\right)\left(x_{3}, 0.6\right)\left(x_{4}, 0.2\right)\left(x_{5}, 0.1\right)\right\}, \\
& C_{3}=\left\{\left(x_{1}, 0.7\right)\left(x_{2}, 0.6\right)\left(x_{3}, 0.5\right)\left(x_{4}, 0.9\right)\left(x_{5}, 0.1\right)\right\}, \\
& C_{4}=\left\{\left(x_{1}, 0.4\right)\left(x_{2}, 0.8\right)\left(x_{3}, 0.6\right)\left(x_{4}, 0.4\right)\left(x_{5}, 0.1\right)\right\} .
\end{aligned}
$$


To evaluate the relative importance of each criterion, the decision-maker must compare pairs of different criteria such as the ones discussed above. In this way the matrix $M$ is obtained:

$M=\left[\begin{array}{llll}1 & \frac{1}{3} & \frac{1}{6} & \frac{1}{5} \\ 3 & 1 & \frac{1}{4} & \frac{1}{3} \\ 6 & 4 & 1 & 2 \\ 5 & 3 & \frac{1}{2} & 1\end{array}\right]$.

From the Eqs. (10)-(12) one can find the maximum eigenvalue at $K_{\max }=4.078783$, and the components of the eigenvector $Q$ are given by

$Q=\left[\begin{array}{l}0.06140 \\ 0.13359 \\ 0.49476 \\ 0.31024\end{array}\right]$.

Using (13),

$W=\left[\begin{array}{c}0.2456 \\ 0.53436 \\ 1.97904 \\ 1.2409\end{array}\right]$.

The decision set can then be represented as

$$
\begin{aligned}
& D=C_{1}^{0.2456} \cap C_{2}^{0.53436} \cap C_{3}^{1.979} \cap C_{4}^{1.2409} \text {, } \\
& C_{1}^{0.2456}=\left\{\left(x_{1}, 0.568\right)\left(x_{2}, 0.946\right)\left(x_{3}, 0.744\right)\right. \\
& \left.\left(x_{4}, 0.843\right)\left(x_{5}, 0.744\right)\right\} \text {, } \\
& C_{2}^{0.53436}=\left\{\left(x_{1}, 0.887\right)\left(x_{3}, 0.761\right)\left(x_{3}, 0.761\right)\right. \\
& \left.\left(x_{4}, 0.423\right)\left(x_{5}, 0.292\right)\right\} \text {, } \\
& C_{3}^{1.979}=\left\{\left(x_{1}, 0.493\right)\left(x_{2}, 0.363\right)\left(x_{3}, 0.253\right)\right. \\
& \left.\left(x_{4}, 0.811\right)\left(x_{5}, 0.0104\right)\right\} \text {, } \\
& C_{4}^{1.2409}=\left\{\left(x_{1}, 0.32\right)\left(x_{2}, 0.758\right)\left(x_{3}, 0.053\right)\right. \\
& \left.\left(x_{4}, 0.320\right)\left(x_{5}, 0.574\right)\right\} \text {, } \\
& D=\left\{\left(x_{1}, 0.32\right)\left(x_{2}, 0.363\right)\left(x_{3}, 0.253\right)\left(x_{4}, 0.320\right)\right. \\
& \left.\left(x_{5}, 0.104\right)\right\} \text {. }
\end{aligned}
$$

Normalizing the set $D$, by dividing each $\mu_{D}\left(x_{i}\right)$ by $\operatorname{Max} \mu_{D}(x)$, we obtain

$$
\begin{aligned}
& D^{*}= \\
& \quad\left\{\left(x_{1}, 0.881\right)\left(x_{2}, 1\right)\left(x_{3}, 0.697\right)\left(x_{4}, 0.879\right)\left(x_{5}, 0.028\right)\right\} .
\end{aligned}
$$

The optimal decision is the one with the greatest degree of membership in $D$. In this case it will be $x_{2}$, workforce training, which has a degree of membership of 0.363 in $D$. The set $D^{*}$ provides a relative ordering of the decision alternatives, that is, it shows a measure of the distance between the optimal decision and the other alternatives. In this regard, we can see that $x_{1}$, purchasing of new machinery, has the nearest distance to $x_{2}$ and, accordingly, is quite capable to meet the stated criteria of becoming a certified supplier.

In the final evaluation of the optimal decision, $x_{2}$, the financial constraints that the decisionmaker faces have had a decisive influence. It is, therefore, concluded that the training program has the potential advantage of complying with this requirement as well as with providing knowledge about certain managerial techniques (e.g., JIT) to be adopted by the company. Alternative actions like the preventive maintenance program (especially, if the installations are in bad shape) or increasing the number of certified vendors do not have an identical fit with the set of stated criteria. Considerations such as the financial constraints or the need of getting some early results to guarantee the long term survival of the company, are the kind of subjective aspects that the decision-maker can introduce in the model.

\section{Concluding remarks}

This paper highligths quality management from a decision-making perspective. Quality definitions are characterized by their inherent ambiguity because of their measurement problems (e.g., excellence, value and exceeding and/or meeting expectations). Alternatively, a quality definition such as conformance to specifications is unable to cope with market changes while it stresses too much internal specifications. Considering the definition of quality as exceeding and/or meeting customer's 
needs, the alternatives to improve quality are characterized by their ambiguity, that is, there are very few alternatives which can be defined as "good" or as "bad" in all cases or under all circumstances. On the contrary, most part of decision alternatives are "more or less good" depending on the particular circumstance being faced by the firm at any given moment. As a result of this ambiguous nature quality decisions should be evaluated under multiple criteria whenever quality is a strategic functional goal. Nevertheless, while the literature recognizes the multiple dimensions of quality [14], not much is said about the interactions between quality decisions and other manufacturing goals. This paper analyzes quality decisions when they are evaluated in the context of the manufacturing strategy, that is, when the impact on costs, flexibility and dependability is also under consideration. In order to model this multiple dimension of quality a multicriteria approach is required.

Fuzzy multicriteria decision theory provides a conceptual framework for the consideration of both ambiguity and multiple dimensions since it allows the introduction of the decision-makers' own experience in the model together with any other relevant information. Thus, the use of fuzzy multicriteria analysis for quality decisions is a correct approach given the partial compatibility of quality decisions and other aspects of manufacturing strategy. Fuzzy set theory offers the possibility of assigning values through the membership function, that is, by the definition of different criteria. This multicriteria method also permits the decision-maker to establish the weights of the different criteria.

There are some managerial techniques that use similar approaches. In particular, Quality Function Deployment (QFD) is characterized by a similar approach [51, pp. 5-11]. It first establishes the critical success factors of the business which resembles the setting of the $C_{j}$ criteria. The relative importance of these critical factors is assessed later and it resembles the process of weighing the different criteria, $w_{j}$. QFD follows from the identification of the different alternatives to reach the already established objectives as it has been done, for example, in this paper with the definition of the $x_{i}$. This phase of the QFD process concludes with the usage of a four-level scale to determine the impact of the alternatives upon the success factors; again, it resembles the definition of the different $\mu_{i j}$. To conclude, this paper introduces a model to approach quality decisions which could help to model techniques such as QFD.

The framework provided in this paper can be easily extended to the analysis of other quality decisions even when these decisions are analyzed from the traditional perspective developed in the quality management literature.

\section{References}

[1] Hofer, C.W. and Schendel, D., 1978. Strategy formulation: Analytical concepts. West Publishing, St. Paul.

[2] Monden, Y., 1982. Toyota Production System. American Institute of Industrial Engineers, New York.

[3] Wheelwright, S., 1984. Manufacturing strategy: Defining the missing link. Strategic Mgmt. J., 5(1): 77-91.

[4] Zadeh, L., 1965. Fuzzy sets. Inform. and Control, 8: $338-353$.

[5] Reeves, C.A. and Bednar, D.A., 1994. Defining quality: Alternatives and implications. Acad. Mgmt. Rev., 19(3); 419-445.

[6] Oakland, J.S., 1989. Total Quality Management. Heinemann, Oxford.

[7] Takeuchi, H. and Quelch, J.A., 1983. Quality is more than making a good product. Har. Bus. Rev., 61(4): 139-145.

[8] Garvin D.A., 1987. Competing on the eight dimensions of quality, Har. Bus. Rev. (November-December): 65: 101-109.

[9] Saraph, J.V., Benson, P.G. and Schroeder, R.G., 1989. An instrument for measuring the critical factors of quality management. Dec. Sci., 20(4): 810-829.

[10] Chan, Y.L. and Yuan, Y., 1990. Dealing with fuzziness in cost-volume-profit analysis. Acc. Bus. Res., 20(78): 83-95.

[11] Hintz, G.W. and Zimmermann, H.J., 1989. A method to control flexible manufacturing systems. Eur. J. Oper. Res., 41(3): 321-334.

[12] Zebda, A., 1984. The investigation of cost variances: A fuzzy set theory approach. Dec. Sci., 15(3): 359-388.

[13] Zebda, A., 1991. The Problem of Ambiguity and Vagueness in Accounting. Beh. Res. Acc., 3: 117-143.

[14] Garvin, D.A., 1988. Managing Quality. Free Press, New York.

[15] ISO 8402, 1986. Quality Vocabulary. British Standards Institution, London.

[16] Ishikawa, K., 1985. What is Total Quality Control? Prentice Hall, Englewood-Cliffs, NJ.

[17] Deming, W., 1982. Quality, Productivity and Competitive Position. MIT Center for Advanced Engineering Study, Cambridge.

[18] Albright, T.L. and Roth, H.P., 1993. Controlling quality on a multidimensional level. J. Cost Mgmt., 2: 29-38. 
[19] Richa, C.E. and Edwards, D.K., 1992. A JIT implementation plan using policy deployment. Manuf. Rev., 5(3): 166-174.

[20] Feigenbaum, A.V., 1986. Quality: The strategic business imperative. Quality Progress (February): 26-30.

[21] Mortyboys, R.J., 1990. Quality management for the 1990s, in: B.G. Dale and J.J. Plunkett. (Eds.), Managing Quality, Philip Allan, New York, pp. 33-43.

[22] Phillips, L.W., Chang, D.R, and Buzzell, R.D., 1983. Product quality, cost position and business performance: $A$ test of some key hypotheses. J. Marketing, 47(2): 26-43.

[23] Schmenner, R.W., 1988. The merit of making things fast. Sloan Mgmt. Rev., 30(1): 11-17.

[24] Gupta, M.M. and Qi, J., 1991. Theory of t-norms and fuzzy inference methods. Fuzzy Sets and Systems 431-450.

[25] Dubois, D. and Prade, H., 1986. New results about properties and semantics of fuzzy set-theoretic operators, in: P.P. Wang and S.K. Chang (Eds.), Fuzzy Sets. Plenum Press, New York, pp. 59-75.

[26] Alsina, C., Trillas, E. and Valverde, L., 1983. On some logical connectives for fuzzy set theory. J. Math. Anal. Appl., 15-26.

[27] Bellman, R.E. and Zadeh, L.A., 1970. Decision making in a fuzzy environment. Mgmt. Sci., 17(4): 141-164.

[28] Zeleny, M., 1982. Multiple Criteria Decision Making. McGraw Hill, New York.

[29] Wallenius, J., 1973. Comparative evaluation of some interactive approaches to multicriteria optimization. Mgmt. Sci., 21: 1387-1396.

[30] Zimmermann, H.J., 1991. Fuzzy Set Theory and Its Applications. Kluwer, Boston.

[31] Zimmermann, H.J., 1986. Fuzzy set theory and mathematical programming, in: A. Jones et al. (Eds.) Fuzzy Set Theory and Applications. Reidel, Dordrecht. pp. 99-114.

[32] Zimmermann, H.J., 1978. Fuzzy programming and linear programming with several objective functions. Fuzzy Sets and Systems, 1(1): 45-55.

[33] Yager, R.R., 1978. Fuzzy decision making including unequal objectives. Fuzzy Sets and Systems, 1(1): 87-95.

[34] Baas, M.S. and Kwakernaak, H., 1977. Rating and ranking of multiple-aspect alternatives using fuzzy sets. Automatica, 13: 47-58.

[35] Buckley, J.J., 1985. Ranking alternatives using fuzzy numbers. Fuzzy Sets and Systems, 15(1): 21-31.
[36] Eldukair, Z.D. and Ayyub, B.B., 1992. Multi-attribute fuzzy decisions in construction strategies. Fuzzy Sets and Systems, 46: 155-165.

[37] Saaty, T.L., 1978. Exploring the interface between hierarchies, multiple objectives and fuzzy Sets. Fuzzy Sets and Systems, 1(1): 57-68.

[38] Saaty, T.L., 1980. The Analytic Hierarchy Process. McGraw Hill, New York.

[39] Federov, V.V., Kuz'min, V.B. and Vereskov, A.I., 1982. Membership degrees determination from Saaty matrix totalities, in: M.M. Gupta and E. Sanchez (Eds)., Approximate Reasoning in Decision Analysis. North-Holland, Amsterdam, New York, pp. 23-30.

[40] Triantaphyllov, E. and Mann, S.H., 1990. An evaluation of the eigenvalue approach for determinig the membership values in fuzzy sets. Fuzzy Sets and Systems, 35: 295-301.

[41] Dyer, J.S., 1990. Remarks on the analytic hierarchy process. Mgmt. Sci., 36(3): 249-258.

[42] Howard, R.A., 1992. Heathens, heretics, and cults: The religious spectrum of decision aiding. Interfaces, 22(6): 15-27.

[43] Harker, P.T. and Vargas, L.G., 1990. Reply to "Remarks on the analytic hierarchy proces". Mgmt. Sci., 36(3): 269-273.

[44] Saaty, T.L., 1990. An exposition of the AHP in reply to the paper "Remarks on the analytic hierarchy process". Mgmt. Sci., 36(3): 259-268.

[45] De Young, G., 1990. Does quality pay?, CFO, September: 24-34.

[46] Fine, C., 1985. Managing quality: a comparative assessment. Booz-Allen Manufacturing Issues, New York.

[47] Hay, E.J., 1988. The Just-In-Time Breakthrough: Implementing the New Manufacturing Basics. Wiley, New York.

[48] Noori, H., 1990. Managing the Dynamics of New Technology. Prentice Hall. New Jersey.

[49] Gunn, T.G., 1992. 21st Century Manufacturing: Creating Winning Business Performance. Harper Business. New York.

[50] ASQC Committee, 1990. Principles of Quality Costs. American Society of Quality Control Press, Milwaukee.

[51] Akao, Y. ed., 1990. Quality Function Deployment: Integrating Customer Requirements into Product Design. Productivity Press, Cambridge, MA. 